\title{
Stress-Induced Subclinical Reactivation of Varicella Zoster Virus in Astronauts
}

Satish K. Mehta, Randall J. Cohrs, Bagher Forghani, Gary Zerbe, Donald H. Gilden and Duane L. Pierson

Enterprise Advisory Services Inc. (SKM) and National Aeronautics and Space Administration (DLP), Lyndon B. Johnson Space Center, Houston, Texas; Viral and Rickettsial Disease Laboratory Section of the California Department of Health Services (BF), Richmond, California; Departments of Preventive Medicine and Biometrics (GZ), Neurology (RJC, DHG) and Microbiology (DHG), University of Colorado Health Sciences Center, Denver, Colorado, USA

Corresponding Author: D. L. Pierson, Ph.D., Habitability and Environmental Factors Office, Mail Code SF24, NASA Johnson Space Center, Houston, TX 77058; Tel: 281-483-7166; Fax: 281-483-3058; e-mail: dpierson@ems.jsc.nasa.gov

Running Head: Subclinical VZV reactivation

Key Words: varicella-zoster virus, VZV reactivation, DNA, PCR, saliva, antibody 
Abstract. After primary infection, varicella-zoster virus (VZV) becomes latent in ganglia. VZV reactivation occurs primarily in elderly individuals, organ transplant recipients, and patients with cancer and AIDS, correlating with a specific decline in cellmediated immunity to VZV. VZV can also reactivate after surgical stress. To determine whether VZV can also reactivate after acute non-surgical stress, we examined total DNA extracted from 312 saliva samples of eight astronauts before, during and after space flight for VZV DNA by PCR: 112 samples were obtained 234 to 265 days before flight, 84 samples on days 2 through 13 of space flight, and 116 samples on days 1 through 15 after flight. Before space flight only one of the 112 saliva samples from a single astronaut was positive for VZV DNA. In contrast, during and after space flight, 61 of $200(30 \%)$ saliva samples were positive in all 8 astronauts. No VZV DNA was detected in any of 88 saliva samples from 10 healthy control subjects. These data indicate that VZV can reactivate subclinically in healthy individuals after acute stress. 
Introduction. Primary VZV infection typically causes childhood varicella (chickenpox), after which virus becomes latent in cranial nerve, dorsal root and autonomic ganglia along the entire human neuraxis. Virus reactivates primarily in elderly individuals, as well as in organ transplant recipients and in patients with cancer and AIDS.

Reactivation correlates with a specific decline in cell-mediated immunity to VZV. Although VZV also reactivates after the stress of surgery, including orofacial surgery, the relationship of $V Z V$ reactivation to non-surgical stress has not been studied.

Space flight provides a model of acute, non-surgical stress, involving a unique and unfamiliar environment, together with sudden gravitational changes associated with launch and landing, and the critical requirement for precise actions. In fact, measurable acute stress responses, such as increased urinary cortisol and interleukin-6 levels, have been demonstrated during launch and reentry (Stein and Schluter, 1994). Here, we describe our analysis of saliva samples from eight astronauts (three missions) before, during and after space flight for evidence of VZV reactivation. The samples were coded for blinded polymerase chain reaction (PCR) analysis for VZV DNA in two separate laboratories. We also collected serum samples and compared the anti-VZV IgG titer of astronauts to matched controls. 


\section{MATERIALS AND METHODS}

Subjects. Eight astronauts (seven men and one woman), 37 to 51 years of age (mean, $42.7 \mathrm{yr}$ ) during three space shuttle missions, 10 to 12 days in duration were studied. Before flight, all astronauts were seropositive for VZV. Ten healthy individuals in the same age range served as controls. The study was approved by the Johnson Space Center Institutional Review Board.

Sample Collection. Saliva samples were collected from each astronaut, before, during and after flight, on cotton rolls (Salivette kits, Sarstedt Inc., Newton, NC), stored in polypropylene vials containing $1.0 \mathrm{ml}$ storage buffer ( $1 \%$ sodium dodecyl sulfate, $10 \mathrm{mM}$ Tris-HCl, pH 8.0, $1 \mathrm{mM}$ EDTA), and coded for blinded PCR analysis as described (Payne et al., 1999). Baseline (preflight) samples were collected on arising every other day for 1 month, beginning approximately 6 months before launch. Daily in-flight saliva samples were collected throughout the mission. Post-flight samples were collected on landing day and then daily for 2 weeks. Control saliva samples were collected every other day for 4 weeks. Blood samples were collected from 10 days before flight and 2 to 3 hours after landing. Two blood samples were collected 22 days apart from five control subjects. This collection schedule closely approximated the pre- and post-flight collection schedule during a 12-day flight. Serum was obtained and stored at $-70^{\circ} \mathrm{C}$ until processed.

DNA Extraction and PCR. Saliva specimens were concentrated with a Microsep 100K filtration unit (Filtron Technology Corporation, Northborough, MA), and DNA was extracted with nonorganic extraction reagents (Qiagen Inc., Chatsworth, CA). Microcarrier gel (Molecular Research Center, Inc., Cincinnati, $\mathrm{OH}$ ) was added to facilitate DNA recovery (Boehringer Mannheim, Indianapolis, IN). DNA was dissolved in $50 \mu \mathrm{l}$ of nuclease-free water (Amresco, Solon, $\mathrm{OH}$ ).

Qualitative nested PCR was performed on a 1/25 (2 $\mu \mathrm{l})$ of the extracted DNA using VZV ORF 63-specific primers (Cohrs et al., 1996). Amplified DNA was resolved on agarose gels, stained with ethidium bromide and the image was captured digitally. Quantitative real-time PCR was performed in a TaqMan 7700 sequence detector (Perkin Elmer Biosystems) using fluorescence-based simultaneous amplification and product detection. Primers and probes for VZV gene 63 and for glyceraldehyde 6-phosphate dehydrogenase (GAPdH) DNA sequences were as described (Cohrs et al., 2000).

Antiviral Antibody Assay. IgG antibody against VZV was determined by enzyme immunoassay (EIA) (Forghani et al., 1986). Each serum sample was tested at a 1:200 dilution against viral antigen (VA), a lysate prepared from VZV-infected human diploid lung cells, and against control antigen (CA), a lysate prepared from uninfected cells at the same passage level. Data generated by ElA were expressed as optical density index (ODI), defined as the absorbance value of VA minus the absorbance value of CA divided by the cut-off $(\mathrm{CO})$ value. $\mathrm{CO}$ is defined as the mean absorbance of a large number of VZV-seronegative samples. An ODI of $\geq 1.0$ was considered positive for the 
presence of VZV-specific IgG. An ODI of $<1.0$ was considered negative for the presence of $\mathrm{VZV}$-specific IgG, and may indicate no prior exposure to the virus.

Statistical Analysis. PCR results were analyzed using logistic regression with random subject effects via SAS Proc NLMIXED (SAS, 1999) to determine outcome significance. Assuming independence and binominal distribution, the probability of detecting VZV DNA was determined by the following formula:

$$
P_{i j k}=\frac{e^{\mu+L_{i}+T_{j}+(L T)_{i j}+s_{k}}}{1+e^{\mu+L_{i}+T_{j}+(L T)_{j i}+s_{k}}}
$$

where $P_{i j k}$ is the probability of VZV DNA detection, $L_{i}$ is the detection method ( $i=1$ for nested PCR, $i=2$ for real-time $P C R), T_{j}$ is the effect of the time $(j=1$ preflight, $j=2$ for during flight, $j=3$ for post-flight), and $S_{k}$ identifies the $i^{\text {th }}$ subject. The $(L T)_{i j}$ terms are detection method by time interactions.

\section{Results}

Frequency of VZV DNA detection in saliva before, during and after space flight. Nested PCR analyses was performed on DNA extracted from 312 saliva samples from eight astronauts. Figure $1 \mathrm{~A}$ shows a representative gel after amplification of 40 saliva samples; 13 were positive for VZV DNA. Serial $\log _{10}$ dilutions of purified VZV DNA revealed that nested PCR could detect one copy of target VZV DNA. Figure 1B shows representative results of the real-time PCR assay. Regression analysis indicated that the PCR assay was linear $\left(R^{2}=0.96\right)$ over a $\log _{10}$ range of VZV DNA from $10^{1}$ to $10^{4}$ copies.

Table 1 summarizes the PCR detection of VZV DNA in saliva samples taken before, during, and after space flight. Yellow rectangles indicate samples negative for VZV DNA by both techniques, nested and real time PCR. Green rectangles indicate saliva samples positive for VZV DNA by nested PCR, but negative by real time PCR. Blue rectangles indicate saliva samples positive for VZV DNA by real time PCR, but negative by nested PCR. Red rectangles indicate saliva samples positive for VZV DNA by both techniques, nested and real time PCR. Numbers within blue and red rectangles denote the VZV DNA copy number determined by real time PCR. Before flight, all 112 samples (14 from each of eight astronauts over a 6-month period) were negative for VZV DNA by real-time PCR, while nested PCR detected VZV DNA in one sample from a single subject. Of 84 saliva samples collected from the eight astronauts during flight, 12 samples (14\%) revealed VZV DNA in nested PCR assays, while 14 samples (16\%) were positive for VZV DNA in real-time PCR; 4 saliva samples from three astronauts were positive for VZV DNA in both real-time and nested PCR. Overall, VZV DNA was detected in six of the eight astronauts $(75 \%)$ from 23 of $84(27 \%)$ saliva samples obtained during flight. Analysis of a total of 116 saliva samples collected daily for 15 days after flight revealed VZV DNA in 23 (19\%) samples by nested-PCR, and in 20 $(17 \%)$ samples by real-time PCR; 4 samples from four astronauts were positive by both real-time and nested PCR. Thus, 38 of $116(32 \%)$ saliva samples were positive for VZV 
DNA in all eight (100\%) astronauts for 2 weeks after landing. None of 88 saliva samples collected from 10 healthy control subjects was positive for VZV DNA by either nested or real-time PCR.

Technique (nested PCR vs. real-time PCR) did not affect the detection of VZV DNA either additively $(p=0.1671)$ or interactively $(p=0.441)$. However, the frequency of detection of VZV DNA was significantly greater during and after flight than before flight whether nested PCR ( $p<0.0098$ during and $p<0.0060$ after). The copy number of GAPdH DNA did not differ significantly in the saliva samples collected from astronauts before (22 \pm 6.3 , mean, \pm SEM), during $(21.5 \pm 5.9)$ and after space flight $(20.9 \pm 4.9)$ or in control subjects $(22.5 \pm 4.3)$ by real-time PCR (ANOVA, $p=0.925)$.

Serum anti-VZV IgG in control subjects and astronauts. Serum anti-VZV IgG ODI were determined in five control subjects and in six astronauts 10 days before flight and again 2 to 3 hours after landing. The ODI for one control serum sample was $<1$ indicating no previous exposure to VZV, and therefore was dropped from the analysis. In the remaining 8 samples from 4 control subjects, the average anti-VZV IgG ODI was $5.8 \pm 0.5$, as compared with $14.9 \pm 1.1$ in 12 serum specimens from six astronauts. After accounting for two samples on each subject via analysis of variance, the average anti-VZV IgG ODI for astronauts $(14.9 \pm 1.1, \mathrm{k}=2$ samples on each of $\mathrm{n}=6$ astronauts) was significantly higher ( $F$-test, $p=0.01$ ) than the average IgG ODI for controls $(5.8 \pm$ $0.5, k=2$ samples on each of $n=4$ controls). In each of the six astronauts and 5 controls, there was no significant difference in the anti-VZV IgG titer 10 days before flight compared to that after landing. Unfortunately, serum samples from astronauts weeks to months before or after space flight were not available for analysis.

\section{Discussion}

Using a convenient, non-invasive method of detecting amplifiable VZV DNA from human saliva, this study showed an association between VZV reactivation and acute, nonsurgical stress in healthy individuals. Detection of VZV DNA in saliva has been used previously to establish an association between VZV reactivation and facial palsy, Ramsay Hunt syndrome (facial palsy and zoster oticus) and zoster sine herpete (pain without rash) (Furuta et al., 2000a; 2000b; 2001). In those disorders, real-time PCR revealed considerable day-to-day variation in VZV DNA copy number, consistent with our findings in the astronauts examined. In fact, the low concordance between our nested PCR and real-time PCR results, based on a correlation coefficient of 0.16 , might reflect sampling variation, low VZV DNA copy number or loss of DNA during storage or shipment. However, PCR results grouped according to time of sampling (before, during and after flight) revealed a high concordance between the two PCR methods.

Of interest, we also found a two to three-fold increase in circulating anti-VZV IgG in astronauts compared to control subjects. Because we were unable to obtain a second serum specimen from astronauts weeks to months after space flight, it was not possible to demonstrate a significant change in antibody to VZV. For example, a four-fold rise 
and fall in antibody to VZV has been used as evidence of subclinical VZV reactivation in both immunocompromised and immunocompetent individuals (Arvin et al., 1983; Gershon et al., 1984; Schunemann et al., 1998). However, the combination of VZV DNA in saliva and a higher specific antibody response in serum of the astronauts compared to control subjects further confirms subclinical reactivation of VZV.

Two other human herpesviruses, cytomegalovirus and Epstein-Barr virus, have also been shown to reactivate during and after space flight (Mehta et al., 2000; Payne et al., 1999). Although the mechanism by which herpesviruses reactivate during space flight is unknown, the combination of gravitational changes and a confined, unfamiliar environment (extreme isolation from family in a crowded environment, lack of privacy, sleep depravation, micro-gravity, physical and physiological stress) has been associated with increased levels of ACTH and cortisol during and after space flight (Stowe et al., 2000; 2001a; 2001b). Multiple clinical reports also support a role for steroids in zoster. For example, a steroid-dependent asthmatic patient who received two courses of high-dose intravenous methylprednisolone at a time when she was exposed to chickenpox developed classic varicella with complications (Silk et al., 1988). Another study reported four patients who received steroids and developed zoster with an average incubation period of 18 days (Rado et al., 1965). Further, in a study of six zoster patients with preherpetic neuralgia (prolonged pain before rash), two who had been taking long-term, low-dose steroids developed disseminated skin lesions with neurologic complications of zoster paresis and a fatal zoster encephalitis, respectively (Gilden et al., 1991). Finally, steroid therapy in the week before or after VZV vaccination has been associated with an increased incidence of varicella rash (Lydick et al., 1989). While additional experiments are needed to determine how stress-induced hormonal changes lead to human herpesvirus reactivation, this study adds VZV to the list of human herpesviruses that reactivate in response to acute nonsurgical stress. 


\section{ACKNOWLEDGMENTS}

This work was supported in part by NASA grants 111-30-10-03 and 111-30-10-06, the NASA Mir Program, a National Research Council Senior Research Associateship (to SKM), and National Institutes of Health grants AG 06127 (DHG) and NS 32623 (DHG and RJC). We thank C. Essman and J. Wischer for expert technical assistance, the staffs of Viral Immunoserology Section for performing the antiviral antibody assays, Marina Hoffman and Jane Krauhs of Wyle Laboratories Life Sciences Systems for editing and Cathy Allen for manuscript preparation. 


\section{REFERENCES}

Arvin AM, Koropchak CM, Wittek AE. 1983. Immunologic evidence of reinfection with varicella-zoster virus. J Infect Dis 148:200-205.

Cohrs RJ, Barbour M, Gilden DH. 1996. Varicella-zoster virus (VZV) transcription during latency in human ganglia: detection of transcripts mapping to genes $21,29,62$, and 63 in a cDNA library enriched for VZV RNA. J Virol 70:2789-2796.

Cohrs RJ, Randall J, Smith J, Gilden DH, Dabrowski C, van Der Keyl H, Tal-Singer R. 2000. Analysis of individual human trigeminal ganglia for latent herpes simplex virus type 1 and varicella-zoster virus nucleic acids using real-time PCR. J Virol 74:1146411471.

Forghani B. 1986. Varicella-zoster virus antibody. In: Bergmeyer H, ed., Methods of Enzymatic Analysis, Vol 10, $3^{\text {rd }}$ edition, Weinheim: Verlag Chemic, pp 267-284.

Forghani B. 2000. Laboratory diagnosis of Infection. In: Arvin AM and Gershon AA, eds., Varicella-Zozter Virus, Virology and Clinical Management, Cambridge, Cambridge University Press, pp351-382.

Furuta Y, Ohtani F, Fukuda S, Inuyama Y, Nagashima K. 2000a. Reactivation of varicella-zoster virus in delayed facial palsy after dental treatment and oro-facial surgery. J Med Virol 62:42-45.

Furuta Y, Ohtani F, Kawabata H, Fukuda S, Bergstrom T. 2000b. High prevalence of varicella-zoster virus reactivation in herpes simplex virus-seronegative patients with acute peripheral facial palsy. Clin Infect Dis 30:529-533.

Furuta Y, Ohtani F, Sawa H, Fukuda S, Inuyama Y. 2001. Quantitation of varicellazoster virus DNA in patients with Ramsay Hunt syndrome and zoster sine herpete. J Clin Microbiol 39:2856-2859.

Gershon AA, Steinberg SP, Gelb L. 1984. Clinical reinfection with varicella-zoster virus. J Infect Dis 149:137-142.

Gilden DH, Dueland AN, Cohrs R, Martin JR, Kleinschmidt-DeMasters BK, Mahalingam R. 1991. Preherpetic neuralgia. Neurology 41:1215-1218.

Lydick E, Kuter BJ, Zajac BA, Guess HA. 1989. Association of steroid therapy with vaccine-associated rashes in children with acute lymphocytic leukaemia who received Oka/Merck varicella vaccine. NIA Varicella Vaccine Collaborative Study Group. Vaccine 7:549-553. 
Mehta SK, Stowe RP, Feiveson AH, Tyring SK, Pierson DL. 2000. Reactivation and shedding of cytomegalovirus in astronauts during spaceflight. J Infect Dis 182:17611764.

Payne DA, Mehta SK, Tyring SK, Stowe RP, Pierson DL. 1999. Incidence of EpsteinBarr virus in astronaut saliva during spaceflight. Aviat Space Environ Med 70:12111213.

Rado JP, Tako J, Geder L, Jeney E. 1965. Herpes zoster house epidemic in steroidtreated patients. Arch Intern Med 116:329-335.

SAS Institute Inc. 1999. The NLMIXED Procedure. In: SAS/STAT User's Guide, Version 8. Cary, NC:SAS Institute Inc., pp 2419-2504.

Schunemann S, Mainka C, Wolff MH. 1998. Subclinical reactivation of varicella-zoster virus in immunocompromised and immunocompetent individuals. Intervirology 41:98102.

Silk HJ, Guay-Woodford L, Perez-Atayde AR, Geha RS, Broff MD. 1988. Fatal varicella in steroid-dependent asthma. J Allergy Clin Immunol 81:47-51.

Stein TP, Schluter MD. 1994. Excretion of IL-6 by astronauts during spaceflight. Am J Physiol 266:E448-452.

Stowe RP, Mehta SK, Ferrando AA, Feeback DL, Pierson DL. 2001a. Immune responses and latent herpesvirus reactivation in spaceflight. Aviat Space Environ Med 72:884-891.

Stowe RP, Pierson DL, Barrett ADT. 2001b. Elevated stress hormone levels relate to Epstein-Barr virus reactivation in astronauts. Psychosom Med 63:891-895.

Stowe RP, Pierson DL, Feeback DL, Barrett AD. 2000. Stress-induced reactivation of Epstein-Barr virus in astronauts. Neuroimmunomodulation 8:51-58. 


\section{Figure legend}

Figure 1. Detection of VZV DNA in astronaut saliva. Total DNA was extracted from the saliva of astronauts and 312 samples were coded for blinded PCR analysis. Panel A shows a representative gel after nested-PCR for VZV DNA in 40 saliva samples. The presence of VZV DNA is demonstrated by the amplification of a 300-bp VZV-specific DNA fragment (arrow). Each set of PCRs contained serial $\log _{10}$ dilutions of purified VZV DNA, indicating that nested PCR could detect $1\left(\log _{10} 0\right)$ copy of VZV DNA. Negative controls without template DNA (No DNA) were always negative for VZV DNA. Panel $B$ shows a representative graph of results from real time PCR analysis of 20 saliva DNA samples. Each real-time PCR contained serial $\log _{10}$ dilutions of purified VZV DNA (filled circles) that were used to quantitate the VZV DNA in saliva (open circles). The number of cycles required to detect VZV DNA within each sample (threshold cycle) was inversely related to the initial amount of VZV DNA present in the sample. Real-time PCR assay is linear (regression analysis, $R^{2}=0.96$ ) over a range of $\log _{10}$ VZV DNA from $10^{1}$ to $10^{4}$ copies. Reaction mixtures containing no template DNA were always negative for VZV DNA (not shown).

\section{Table 1 legend.}

Yellow rectangles indicate samples negative for VZV DNA by both techniques, nested and real time PCR. Green rectangles indicate saliva samples positive for VZV DNA by nested PCR, but negative by real time PCR. Blue rectangles indicate saliva samples positive for VZV DNA by real time PCR, but negative by nested PCR. Red rectangles indicate saliva samples positive for VZV DNA by both techniques, nested and real time PCR. White rectangles indicate days for which no saliva sample was available (n.a., not available). Numbers within blue and red rectangles denote the VZV DNA copy number determined by real time PCR. VZV DNA was detected in $1 \%$ of 112 samples collected before flight, $27 \%$ of 84 samples collected during flight, and $33 \%$ of 116 samples collected after flight. 
Table 1. Frequency of VZV DNA in astronaut saliva before, during and after space flight (summary of nested and real-time PCR results).

\begin{tabular}{|c|c|c|c|c|c|c|c|c|c|}
\hline & & \multicolumn{8}{|c|}{ Subject } \\
\hline & Days & 1 & 2 & 3 & 4 & 5 & 6 & 7 & 8 \\
\hline \multirow{14}{*}{$\begin{array}{l}\text { Before } \\
\text { flight }\end{array}$} & 265 & & & & & & & & \\
\hline & 263 & & & & & & & & \\
\hline & 261 & & & & & & & & \\
\hline & 259 & & & & & & & & \\
\hline & 255 & & & & & & & & \\
\hline & 253 & & & & & & & & \\
\hline & 249 & & & & & & & & \\
\hline & 247 & & & & & & & & \\
\hline & 243 & & & & & & & & \\
\hline & 241 & & & & & & & & \\
\hline & 239 & & & & & & & & \\
\hline & 237 & & & & & & & & \\
\hline & 236 & & & & & & & & \\
\hline & 234 & & & & & & & & \\
\hline \multirow{12}{*}{$\begin{array}{l}\text { During } \\
\text { flight }\end{array}$} & 2 & & & & & 110 & & & \\
\hline & 3 & & & & & & $<10$ & & \\
\hline & 4 & & & & & & & & \\
\hline & 5 & & & 24 & & & & & \\
\hline & 6 & & & & & 47 & $<10$ & & \\
\hline & 7 & & & & & & $<10$ & & \\
\hline & 8 & & & & & & $<10$ & & \\
\hline & 9 & $<10$ & & 110 & & & $<10$ & & \\
\hline & 10 & & & & & 25,000 & $<10$ & & \\
\hline & 11 & \multirow{3}{*}{\multicolumn{4}{|c|}{ no sample available, flight terminated }} & & & & $<10$ \\
\hline & 12 & & & & & $<10$ & & & \\
\hline & 13 & & & & & & & & \\
\hline \multirow[t]{15}{*}{ After flight } & 1 & & $<10$ & n.a. & & $<10$ & 220 & & \\
\hline & 2 & & n.a. & & & $<10$ & n.a. & & \\
\hline & 3 & & & & & n.a. & 6800 & & \\
\hline & 4 & & & & & 330 & & & \\
\hline & 5 & & & & & & 24 & & \\
\hline & 6 & & $<10$ & 800 & & & & & $<10$ \\
\hline & 7 & & & & & 190 & & & 55 \\
\hline & 8 & & & & & 280 & & & 600 \\
\hline & 9 & & & & & & & & 5500 \\
\hline & 10 & & & & & 2100 & & & \\
\hline & 11 & & & 6000 & & & & & \\
\hline & 12 & & & & & 440 & & & \\
\hline & 13 & & & & & & & & \\
\hline & 14 & & & & & & & $<10$ & \\
\hline & 15 & & & & & & & & \\
\hline
\end{tabular}

